\title{
Reliable $H_{\infty}$ Filtering for Stochastic
}

\section{Spatial-Temporal Systems with Sensor Saturations and Failures}

\author{
Dong Wang ${ }^{a}$, Bo Shen ${ }^{a, *}$, Zidong Wang ${ }^{b, c}$, Fuad E. Alsaadi ${ }^{c}$ and Abdullah M. Dobaie ${ }^{c}$
}

\begin{abstract}
This paper is concerned with the reliable $H_{\infty}$ filtering problem for a class of stochastic spatial-temporal systems with sensor saturations and failures. Different from the continuous-spatial temporal systems, the dynamic behaviour of the system under consideration evolves in a discrete rectangular region. The aim of this paper is to estimate the system states through the measurements received from a set of sensors located in some specified points. In order to cater for more realistic signal transmission process, the phenomena of sensor saturations and sensor failures are taken into account. By using the vector reorganization approach, the spatial-temporal system is first transformed into an equivalent ordinary differential dynamic system. Then, a filter is constructed and a sufficient condition is obtained under which the filtering error dynamics is asymptotically stable in probability and the $H_{\infty}$ performance requirement is met. Based on the analysis results, the desired reliable $H_{\infty}$ filter is designed. Finally, an illustrative example is given to show the effectiveness of the proposed filtering scheme.
\end{abstract}

\section{Keywords}

Distributed parameter systems; estimation and filtering; $H_{\infty}$ control and estimation; nonlinear systems; stochastic systems.

\section{INTRODUCTION}

The $H_{\infty}$ filtering problem can be briefly described as the design of a filter to estimate the unknown state by using the available measurements such that the $\mathcal{L}_{2}$ gain from the exogenous disturbance to the estimation error is less than a prescribed level [1]. Different from the well-known Kalman filtering where the statistical properties of the noise processes are required to be known exactly, in the $H_{\infty}$ filtering scheme, the disturbance noises are only assumed to be bounded in energy. Due to such an advantage, in the past years, considerable

This work was supported in part by the the Deanship of Scientific Research (DSR) at King Abdulaziz University in Saudi Arabia under Grant 16-135-35-HiCi, the National Natural Science Foundation of China under Grants 61329301, 61134009 and 61473076, the Shanghai Rising-Star Program of China under Grant 13QA1400100, the Shu Guang project of Shanghai Municipal Education Commission and Shanghai Education Development Foundation under Grant 13SG34, the Fundamental Research Funds for the Central Universities, the DHU Distinguished Young Professor Program, and the Alexander von Humboldt Foundation of Germany.

${ }^{a}$ School of Information Science and Technology, Donghua University, Shanghai 200051, China.

${ }^{b}$ Department of Computer Science, Brunel University London, Uxbridge, Middlesex, UB8 3PH, United Kingdom.

${ }^{c}$ Faculty of Engineering, King Abdulaziz University, Jeddah 21589, Saudi Arabia.

* Corresponding author. Email: shenbodh@gmail.com 
research efforts have been made with respect to $H_{\infty}$ filtering problems and various $H_{\infty}$ filters have been designed in order to cater for specific engineering issues, see e.g. [2-14]. In the design of $H_{\infty}$ filters, the general techniques include the linear matrix inequality approach $[2,3,7,9,10,13]$ and Riccati equation approach $[5,6,11]$.

It is well known that the characteristics of the available measurements play a key role in the filtering problem. The measurements are usually received from the sensors placed according to engineering requirements. In reality, due to physical limitations of the components, sensor saturations often occur. Compared to the saturation-free case, the nonlinear feature induced by sensor saturation brings in significant difficulties in designing a filter that can guarantee desired filtering performance. Therefore, in the past decade, the $H_{\infty}$ filtering problem with sensor saturations has received considerable attention and a number of results have been reported that have offered different ways to handle the sensor saturation phenomenon, see, e.g., [15-22]. For example, both the sensor saturations and the missing measurements have been considered in [18] and the $H_{\infty}$ filter has been designed for systems with such phenomena of incomplete information. In [16], the robust $H_{\infty}$ filtering problem has been investigated for a class of discrete time-varying Markovian jump systems with randomly occurring nonlinearities and sensor saturations.

Another sensor-related imperfection, sensor failure, is often encountered in real-world systems owing to unavoidable component aging. When the sensor failure occurs, the dynamic behavior of the filtering error will not evolve as expected and the overall filtering performance might be seriously degraded. As such, the reliable filtering problem with sensor failures has emerged as an attractive topic of research. So far, a variety of reliable $H_{\infty}$ filters have been proposed to deal with the sensor failures. For example, in [23], a new sensor failure model with uncertainties has been proposed and a robust and reliable $H_{\infty}$ filter has been designed for a class of nonlinear networked control systems by using the T-S fuzzy approach. In [24], an adaptive reliable $H_{\infty}$ filter has been designed against sensor failures by the aid of the linear matrix inequality approach and adaptive method. Very recently, a reliable filter has been designed in [25] for a class of discrete-time piecewise linear systems where both sensor failures and infinite distributed delays are taken into account.

On the other hand, spatial-temporal systems have received increasing research attention because of their successful applications in practical situations such as wildfire, road traffic and flood. With respect to the filtering problems, there have recently appeared some initial results on the stochastic spatial-temporal systems. For example, in [26], a robust $H_{\infty}$ filter has been designed for linear stochastic spatial temporal systems with external disturbance and measurement noise, and the results have been extended in [27] to the nonlinear case and the nonlinear robust $H_{\infty}$ filtering problem has been investigated by recurring to the fuzzy interpolation approach. However, the existing $H_{\infty}$ filtering methods are only applied to continuous-spatial temporal systems. For the discrete-spatial temporal systems with or without sensor saturations and failures, to the best of our knowledge, the corresponding reliable $H_{\infty}$ filtering problem has not been investigated yet. Indeed, for stochastic discrete-spatial temporal systems with imperfect sensors, there would be some fundamental difficulties to be overcome in the design of reliable $H_{\infty}$ filter. For example, how to analyze the complex dynamic 
behavior induced by spatial-temporal nature and how to design the reliable $H_{\infty}$ filter for such spatial-temporal systems when the sensors can't work normally. Therefore, it is the main motivation of this paper to offer satisfactory answers to these questions by developing an $H_{\infty}$ filtering scheme for discrete-spatial temporal systems with both sensor saturations and failures.

In this paper, the reliable $H_{\infty}$ filtering problem is investigated for a class of stochastic spatial-temporal systems with sensor saturations and failures. The dynamic behavior of systems under consideration evolves in both space and time. In order to estimate the system states in the given rectangular region, some sensors are located in the specified points. The effects from both the sensor saturations and the sensor failures are taken into consideration in the information transmission. By using the vector reorganization approach, the spatial-temporal system is represented by an equivalent ordinary differential dynamic system. For the obtained ordinary differential dynamic system, a filter is constructed and the $H_{\infty}$ performance of the filtering error dynamics is analyzed. With the help of the Lyapunov stability theory, a sufficient condition is derived under which the filtering error dynamics is asymptotically stable in probability and the $H_{\infty}$ performance requirement is satisfied. Based on the obtained sufficient condition, the desired reliable $H_{\infty}$ filter is designed. Finally, an illustrative example is presented to show the effectiveness of the filtering scheme proposed.

Notation The notation used here is fairly standard except where otherwise stated. $\mathbb{R}^{n}$ and $\mathbb{R}^{n \times m}$ denote, respectively, the $n$ dimensional Euclidean space and the set of all $n \times m$ real matrices. $\|A\|$ refers to the norm of a matrix $A$ defined by $\|A\|=\sqrt{\operatorname{trace}\left(A^{T} A\right)}$. The notation $X \geq Y$ (respectively, $X>Y$ ), where $X$ and $Y$ are real symmetric matrices, means that $X-Y$ is positive semi-definite (respectively, positive definite). $M^{T}$ represents the transpose of the matrix $M . I$ denotes the identity matrix of compatible dimension. $\operatorname{diag}\{\cdots\}$ stands for a block-diagonal matrix. $\mathbb{E}\{x\}$ stands for the expectation of the stochastic variable $x$. $\mathrm{P}\{\cdot\}$ means the occurrence probability of the event ".". $\mathcal{L}_{2}\left([0, \infty) ; \mathbb{R}^{n}\right)$ is the space of square integrable vector-valued functions. "®" and "०" represent the Kronecker and Hadamard products, respectively. In symmetric block matrices, "*" is used to denote a term induced by symmetry. Matrices, if they are not explicitly specified, are assumed to have compatible dimensions.

\section{Problem Formulation}

Stochastic spatial-temporal systems have been studied in $[26,27]$ where the systems are continuous in both time and space. In this paper, we consider a class of stochastic discrete-spatial temporal systems. Specifically, let a discrete rectangular region be given as $\{0,1,2, \ldots, M\} \times\{0,1,2, \ldots, N\}$ and denote by $(m, n)$ the location of the $m$ th row and $n$th column in the rectangular region. The system under consideration is of the following 
form:

$$
\left\{\begin{aligned}
d x_{m, n}(t)= & \left\{\kappa\left(x_{m+1, n}(t)+x_{m-1, n}(t)+x_{m, n+1}(t)+x_{m, n-1}(t)-4 x_{m, n}(t)\right)\right. \\
& \left.+A\left(x_{m+1, n}(t)+x_{m, n+1}(t)-2 x_{m, n}(t)\right)+B x_{m, n}(t)+G v_{m, n}(t)\right\} d t \\
& +G_{1} x_{m, n}(t) d W_{m, n}(t), \\
y_{m_{i}, n_{i}}(t)= & \sigma\left(C_{m_{i}, n_{i}} x_{m_{i}, n_{i}}(t)\right)+D_{m_{i}, n_{i}} v_{m_{i}, n_{i}}(t), \quad i=1,2, \cdots, l \\
z_{m, n}(t)= & H x_{m, n}(t),
\end{aligned}\right.
$$

where $x_{m, n}(t) \in \mathbb{R}^{n_{x}}$ is the state vector at the location $(m, n), y_{m_{i}, n_{i}}(t) \in \mathbb{R}^{n_{y}}$ is the measurement output received by the sensor at the location $\left(m_{i}, n_{i}\right), z_{m, n}(t) \in \mathbb{R}^{n_{z}}$ is the signal to be estimated at the location $(m, n), v_{m, n}(t) \in \mathbb{R}^{n_{v}}$ is the external disturbance input at the location $(m, n)$ which belongs to $\mathcal{L}_{2}\left([0, \infty) ; \mathbb{R}^{n_{v}}\right)$, and $W_{m, n}(t) \in \mathbb{R}$ is a zero-mean Brownian motion with unit covariance that is used to describe the stochastic noise at the location $(m, n)$. The initial values of states at the inner points are denoted by $x_{m, n}(0)=x_{m, n}^{0}$. $\kappa, A, B, G, G_{1}, C_{m_{i}, n_{i}}, D_{m_{i}, n_{i}}$ and $H$ are known real constant matrices with appropriate dimensions.

The saturation function $\sigma(\cdot)$ is defined as

$$
\sigma(w)=\left[\begin{array}{llll}
\sigma\left(w_{1}\right) & \sigma\left(w_{2}\right) & \ldots & \sigma\left(w_{n_{y}}\right)
\end{array}\right]^{T}, \quad \forall w \in \mathbb{R}^{n_{y}}
$$

where $\sigma\left(w_{i}\right)=\operatorname{sign}\left(w_{i}\right) \min \left\{\underline{w}_{i},|w|_{i}\right\}$ for $i=1,2, \cdots, n_{y}$. Here, "sign" denotes the signum function and $\underline{w}_{i}$ is the saturation level. It is known from [20] that, for the saturation function $\sigma(\cdot)$, there exists scalar $0 \leq a_{i}<1$ such that the following inequality holds:

$$
\left[\sigma\left(w_{i}\right)-a_{i} w_{i}\right]\left[\sigma\left(w_{i}\right)-w_{i}\right] \leq 0
$$

Assumption 1: The values of states on the boundary satisfy the Dirichlet boundary condition, i.e., $x_{m, n}(t)=$ 0 for $m=0, M$ or $n=0, N$.

Remark 1: Spatial-temporal systems have wide applications in the practical engineering such as ocean or wind circulation and heat or propagation equations [28]. Spatial-temporal systems are usually characterized in terms of partial differential equations which are continuous in both time and space. In this paper, we are concerned with a new model to describe spatial-temporal systems where the dynamic behavior evolving in space is discrete, which could be more suitable to model digitally transmitted signals in a dynamical way.

Remark 2: It is not difficult to see from (1) that the states on the boundary do not follow the dynamic evolution characterized by (1) and the values of states on the boundary need to be given. Actually, the boundary condition is of vital importance for the existence of the solution to the spatial-temporal systems. In this paper, the Dirichlet boundary condition is imposed for the spatial-temporal systems under consideration. The spatial-temporal systems with other boundary conditions could be dealt with by following the same lines in this paper.

Reorganize all the state variable $x_{m, n}(t)(m=0,1, \cdots, M$ and $n=0,1, \cdots, N)$ into the following vector

$$
x(t)=\left[x_{0,0}^{T}(t) \ldots x_{m, 0}^{T}(t) \ldots x_{M, 0}^{T}(t) \ldots x_{m, n}^{T}(t) \ldots x_{0, N}^{T}(t) \ldots x_{m, N}^{T}(t) \ldots x_{M, N}^{T}(t)\right]^{T} .
$$


Introduce an index $j$ defined as $j=n(M+1)+m+1$ and set $x_{j}(t)=x_{m, n}(t)$. Obviously, $x_{j}(t)$ is the $j$ th element in the vector given by (4). The index set of boundary points is denoted by $\mathfrak{J}=\{j=$ $n(M+1)+m+1 \mid m=0, n=0,1, \cdots, N$ or $m=M, n=0,1, \cdots, N$ or $m=0,1, \cdots, M, n=0$ or $m=0,1, \cdots, M, n=N\}$. Similarly, we can define $v(t), z(t), v_{j}(t), W_{j}(t)$ and $z_{j}(t)$. Moreover, the $l$ sensors fitted in the rectangular region are organized as $y(t)=\left[\begin{array}{lllll}y_{m_{1}, n_{1}}^{T}(t) & y_{m_{2}, n_{2}}^{T}(t) & \ldots & y_{m_{l}, n_{l}}^{T}(t)\end{array}\right]^{T}$. Then, the spatial-temporal system (1) can be transformed into the following ordinary differential dynamic system

$$
\left\{\begin{aligned}
d x_{j}(t) & =\left[\kappa \Gamma_{1 j} x(t)+A \Gamma_{2 j} x(t)+B x_{j}(t)+G v_{j}(t)\right] d t+G_{1} x_{j}(t) d W_{j}(t), \\
y(t) & =\sigma(\bar{C} x(t))+\bar{D} v(t), \\
z_{j}(t) & =H x_{j}(t)
\end{aligned}\right.
$$

where

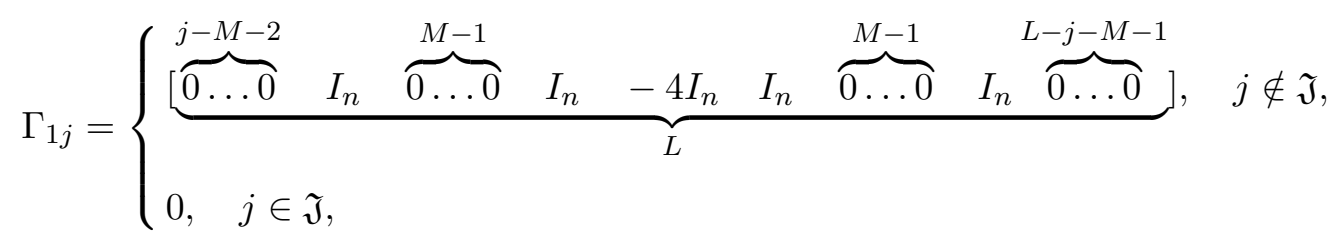

$$
\begin{aligned}
& \Gamma_{2 j}=\left\{\begin{array}{l}
\underbrace{\overbrace{0 \ldots 0}^{j-1}-2 I_{n} \quad I_{n} \quad \overbrace{0 \ldots 0}^{M-1} I_{n} \overbrace{0 \ldots 0}^{L-j-M-1}}_{L}], j \notin \mathfrak{J}, \\
0, \quad j \in \mathfrak{J},
\end{array}\right. \\
& \bar{C}=\left[\begin{array}{llll}
C_{1}^{T} & C_{2}^{T} & \ldots & C_{l}^{T}
\end{array}\right]^{T}, \quad \bar{D}=\left[\begin{array}{llll}
D_{1}^{T} & D_{2}^{T} & \ldots & D_{l}^{T}
\end{array}\right]^{T},
\end{aligned}
$$

with

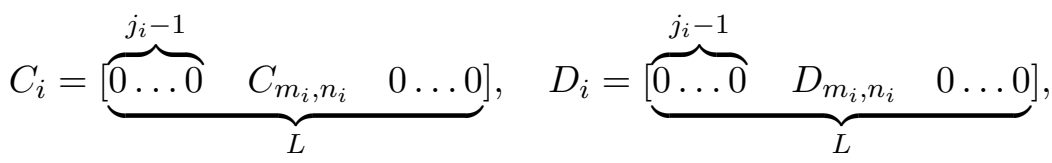

$$
\begin{aligned}
& j_{i}=n_{i}(M+1)+m_{i}+1, \quad L=(M+1)(N+1) .
\end{aligned}
$$

Setting

$$
\begin{aligned}
& \Gamma_{1}=\left[\begin{array}{llll}
\Gamma_{11}^{T} & \Gamma_{12}^{T} & \ldots & \Gamma_{1 L}^{T}
\end{array}\right]^{T}, \quad \Gamma_{2}=\left[\begin{array}{llll}
\Gamma_{21}^{T} & \Gamma_{22}^{T} & \ldots & \Gamma_{2 L}^{T}
\end{array}\right]^{T}, \\
& \bar{A}=\left(I_{L} \otimes \kappa\right) \Gamma_{1}+\left(I_{L} \otimes A\right) \Gamma_{2}+I_{L} \otimes B, \quad J=\left[\begin{array}{llll}
1 & 1 & \ldots & 1
\end{array}\right]^{T}, \\
& \bar{G}=I_{L} \otimes G, \quad G_{1}=I_{L} \otimes G_{1}, \quad \bar{H}=I_{L} \otimes H, \\
& W(t)=\left[\begin{array}{llll}
W_{1}(t) J^{T} & W_{2}(t) J^{T} & \ldots & W_{N}(t) J^{T}
\end{array}\right]^{T},
\end{aligned}
$$

we further have the following augmented system

$$
\left\{\begin{aligned}
d x(t) & =[\bar{A} x(t)+\bar{G} v(t)] d t+\bar{G}_{1} x(t) \circ d W(t) \\
y(t) & =\sigma(\bar{C} x(t))+\bar{D} v(t) \\
z(t) & =\bar{H} x(t)
\end{aligned}\right.
$$


with the initial condition

$$
x(0)=\left[\left(x_{0,0}^{0}\right)^{T} \ldots\left(x_{m, 0}^{0}\right)^{T} \ldots\left(x_{M, 0}^{0}\right)^{T} \ldots\left(x_{m, n}^{0}\right)^{T} \ldots\left(x_{0, N}^{0}\right)^{T} \ldots\left(x_{m, N}^{0}\right)^{T} \ldots\left(x_{M, N}^{0}\right)^{T}\right]^{T}
$$

where $x_{m, n}^{0}=0$ for $m=0, n=0,1, \cdots, N$ or $m=M, n=0,1, \cdots, N$ or $m=0,1, \cdots, M, n=0$ or $m=0,1, \cdots, M, n=N$.

In this paper, we aim to estimate all the states in the given rectangular region by employing the available measurement $y(t)$. When the sensors receive the measurement $y(t)$, the possible sensor failures may occur. Now, we consider the following model to describe such sensor failures:

$$
y^{f}(t)=F y(t)
$$

where $y^{f}(t) \in \mathbb{R}^{n_{y} l}$ is the final measurement entering the filter and $F \in \mathbb{R}^{n_{y} l \times n_{y} l}$ is the sensor fault matrix defined by

$$
F=\operatorname{diag}\left\{f_{1}, f_{2}, \ldots, f_{n_{y} l}\right\}
$$

and $f_{i}$ satisfies $\underline{f}_{i} \leq f_{i} \leq \bar{f}_{i}$ for all $i=1,2, \ldots, n_{y} l$.

For the purpose of manipulation convenience, we rewrite the fault matrix $F$ as follows

$$
F=F_{0}+\Delta F, \quad \Delta F \leq \widetilde{F}
$$

where

$$
\begin{aligned}
& F_{0}=\operatorname{diag}\left\{\frac{f_{1}+\bar{f}_{1}}{2}, \frac{f_{2}+\bar{f}_{2}}{2}, \ldots, \frac{\underline{f}_{n_{y} l}+\bar{f}_{n_{y} l}}{2}\right\}, \\
& \widetilde{F}=\operatorname{diag}\left\{\frac{\bar{f}_{1}-\underline{f}_{1}}{2}, \frac{\bar{f}_{2}-\underline{f}_{2}}{2}, \ldots, \frac{\bar{f}_{n_{y} l}-\underline{f}_{n_{y} l}}{2}\right\} .
\end{aligned}
$$

Based on the measurements with sensor failures, the following filter structure is adopted

$$
\left\{\begin{aligned}
d \hat{x}(t) & =\left[\bar{A} \hat{x}(t)+L_{f} y^{f}(t)\right] d t \\
\hat{z}(t) & =\bar{H} \hat{x}(t)
\end{aligned}\right.
$$

where $\hat{x}(t) \in \mathbb{R}^{n_{x} L}$ is the state estimate, $\hat{z}(t) \in \mathbb{R}^{n_{z} L}$ is an estimate of the output $z(t)$ and $L_{f}$ is the parameter matrix to be determined, $\hat{x}(0)$ is the initial state.

Setting $\eta(t)=\left[\begin{array}{ll}x^{T}(t) & \hat{x}^{T}(t)\end{array}\right]^{T}$ and $e(t)=z(t)-\hat{z}(t)$, we have the following filtering error system:

$$
\left\{\begin{aligned}
d \eta(t) & =\left[\tilde{A} \eta(t)+\tilde{A}_{1} \sigma(\bar{C} Z \eta(t))+\tilde{B} v(t)\right] d t+\tilde{B}_{1} \eta(t) \circ d \bar{W}(t), \\
e(t) & =\tilde{C} \eta(t)
\end{aligned}\right.
$$


with the initial condition $\eta(0)=\left[\begin{array}{ll}x^{T}(0) & \hat{x}^{T}(0)\end{array}\right]^{T}$, where

$$
\begin{aligned}
& \tilde{A}=\left[\begin{array}{ll}
\bar{A} & 0 \\
0 & \bar{A}
\end{array}\right], \quad \tilde{A}_{1}=\left[\begin{array}{c}
0 \\
L_{f} F
\end{array}\right], \quad \tilde{B}=\left[\begin{array}{c}
\bar{G} \\
L_{f} F \bar{D}
\end{array}\right], \quad \tilde{B}_{1}=\left[\begin{array}{cc}
\bar{G}_{1} & 0 \\
0 & 0
\end{array}\right], \\
& \bar{W}(t)=\left[\begin{array}{l}
W(t) \\
W(t)
\end{array}\right], \quad \tilde{C}=\left[\begin{array}{ll}
\bar{H} & -\bar{H}
\end{array}\right], \quad Z=\left[\begin{array}{ll}
I & 0
\end{array}\right] .
\end{aligned}
$$

Note that the augmented system (10) is a stochastic system due to the existence of stochastic process $\bar{W}(t)$. Therefore, we need to introduce the following stochastic stability concept.

Definition 1: [29] The zero solution of the system (10) with $v(t)=0$ is said to be asymptotically stable in probability if, (i) for any $\varepsilon>0, \lim _{\eta(0) \rightarrow 0} \mathrm{P}\left\{\sup _{t \geq 0}\|\eta(t)\|>\varepsilon\right\}=0$ and (ii) for the initial condition $\eta(0)$, $\mathrm{P}\left\{\lim _{t \rightarrow 0}\|\eta(t)\|=0\right\}=1$.

The reliable $H_{\infty}$ filtering problem to be investigated can be now stated as follows. For the stochastic spatial-temporal system (1), we are interested in finding the filter gain matrix $L_{f}$ such that, for all possible sensor saturations and sensor failures, the following two requirements are simultaneously satisfied.

1) The zero solution of the system (10) with $v(t)=0$ is asymptotically stable in probability;

2 ) Under the zero initial condition, the filtering error $e(t)$ satisfies

$$
\mathbb{E}\left\{\int_{0}^{\infty}\|e(t)\|^{2} d t\right\}<\gamma^{2} \int_{0}^{\infty}\|v(t)\|^{2} d t
$$

for all nonzero $v(t)$, where $\gamma>0$ is a given disturbance attenuation level.

Remark 3: In this paper, the vector reorganization approach is employed to transform the spatial-temporal systems into an equivalent ordinary differential dynamic systems. Such a practice can effectively overcome the difficulty resulting from the complex dynamic behavior of the spatial-temporal systems and the corresponding $H_{\infty}$ filtering problem will be readily solved.

\section{Main Results}

In this section, we first derive a sufficient condition under which the filtering error system (10) is asymptotically stable in probability in the case of disturbance-free and, simultaneously, the $H_{\infty}$ performance constraint (12) is met. Based on the obtained condition, the design method of the desired reliable $H_{\infty}$ filter is then given.

Let us start with tackling the saturation function $\sigma$. According to the inequality (3), it is easily known that the nonlinear function $\sigma(\bar{C} Z \eta(t))$ satisfies

$$
[\sigma(\bar{C} Z \eta(t))-\Lambda \bar{C} Z \eta(t)]^{T}[\sigma(\bar{C} Z \eta(t))-\bar{C} Z \eta(t)] \leq 0
$$

where $\Lambda=\operatorname{diag}\left\{a_{1}, a_{2}, \ldots, a_{n_{y}}\right\}$.

The following lemma will be used in the proof of our main theorems.

Lemma 1: [30] Let $J=J^{T}, M$ and $N$ be real matrices of appropriate dimensions with $F$ satisfying $F F^{T} \leq I$. Then $J+M F N+N^{T} F^{T} M^{T}<0$ if and only if there exists a positive scalar $\sigma$ such that 
$J+\sigma^{-1} M M^{T}+\sigma N^{T} N<0$ or, equivalently,

$$
\left[\begin{array}{ccc}
J & M & \sigma N^{T} \\
M^{T} & -\sigma I & 0 \\
\sigma N & 0 & -\sigma I
\end{array}\right]<0
$$

The following theorem provides a sufficient condition that the desired filter should satisfy.

Theorem 1: For the given disturbance attenuation level $\gamma>0$ and the filter parameter matrix $L_{f}$, the origin of the system (10) with $v(t)=0$ is asymptotically stable in probability and, under the zero initial condition, the filtering error $e(t)$ satisfies the $H_{\infty}$ performance constraint (12) for all nonzero exogenous disturbances $v(t)$ if there exist a positive definite matrix $P$ and a positive scalar $\varepsilon_{1}$ satisfying

$$
\Phi=\left[\begin{array}{ccc}
\tilde{A}^{T} P+P \tilde{A}+\tilde{B}_{1}^{T} P \tilde{B}_{1}+\tilde{C}^{T} \tilde{C}-\varepsilon_{1} K_{1} & P \tilde{A}_{1}-\varepsilon_{1} K_{2} & P \tilde{B} \\
* & -\varepsilon_{1} I & 0 \\
* & * & -\gamma^{2} I
\end{array}\right]<0
$$

where $K_{1}=Z^{T} \bar{C}^{T} \Lambda \bar{C} Z$ and $K_{2}=-Z^{T} \bar{C}^{T}(\Lambda+I) / 2$.

Proof: Choose the Lyapunov function as $V(\eta(t))=\eta^{T}(t) P \eta(t)$. With the help of Itô formula, the stochastic derivative of $V(\eta(t))$ along the trajectory of system (10) with $v(t)=0$ is obtained as

$$
\begin{aligned}
\mathbb{L} V(\eta(t))= & \eta^{T}(t)\left(\tilde{A}^{T} P+P \tilde{A}+\tilde{B_{1}^{T}} P \tilde{B}_{1}\right) \eta(t)+\sigma^{T}(\bar{C} Z \eta(t)) \tilde{A}_{1}^{T} P \eta(t) \\
& +\eta^{T}(t) P \tilde{A_{1}} \sigma(\bar{C} Z \eta(t)) \\
= & \zeta^{T}(t) \bar{\Phi} \zeta(t),
\end{aligned}
$$

where

$$
\begin{aligned}
\zeta(t) & =\left[\begin{array}{cc}
\eta^{T}(t) & \sigma^{T}(\bar{C} Z \eta(t))
\end{array}\right]^{T}, \\
\bar{\Phi} & =\left[\begin{array}{cc}
\tilde{A}^{T} P+P \tilde{A}+\tilde{B_{1}^{T}} P \tilde{B_{1}} & P \tilde{A}_{1} \\
* & 0
\end{array}\right] .
\end{aligned}
$$

By taking into account (13), it can be obtained that

$$
\begin{aligned}
\mathbb{L} V(\eta(t)) & \leq \zeta^{T}(t) \bar{\Phi} \zeta(t)-\varepsilon_{1}[\sigma(\bar{C} Z \eta(t))-\Lambda \bar{C} Z \eta(t)]^{T}[\sigma(\bar{C} Z \eta(t))-\bar{C} Z \eta(t)] \\
& =\zeta^{T}(t) \hat{\Phi} \zeta(t)
\end{aligned}
$$

where

$$
\hat{\Phi}=\left[\begin{array}{cc}
\tilde{A}^{T} P+P \tilde{A}+\tilde{B_{1}^{T} P \tilde{B}_{1}-\varepsilon_{1} K_{1}} & P \tilde{A}_{1}-\varepsilon_{1} K_{2} \\
* & -\varepsilon_{1} I
\end{array}\right] .
$$

Noting that inequality (15) implies $\hat{\Phi}<0$, we have

$$
\mathbb{L} V(\eta(t))<0
$$

from which, the asymptotical stability in probability of system (10) with $v(t)=0$ is ensured in terms of the stochastic Lyapunov stability theory [29]. 
In order to show that the filtering error satisfies the $H_{\infty}$ performance constraint under the zero initial condition, we calculate

$$
\begin{aligned}
& \mathbb{E}\left\{\int_{0}^{t_{f}}\|e(t)\|^{2} d t\right\}-\gamma^{2} \int_{0}^{t_{f}}\|v(t)\|^{2} d t \\
= & \mathbb{E}\left\{\int_{0}^{t_{f}}\left[e^{T}(t) e(t)-\gamma^{2} v^{T}(t) v(t)+\mathbb{L} V(\eta(t))\right] d t\right\}-\mathbb{E}\left(\eta^{T}\left(t_{f}\right) P \eta\left(t_{f}\right)\right) \\
\leq & \mathbb{E}\left\{\int_{0}^{t_{f}}\left[e^{T}(t) e(t)-\gamma^{2} v^{T}(t) v(t)+\mathbb{L} V(\eta(t))\right] d t\right\} \\
= & \mathbb{E}\left\{\int _ { 0 } ^ { t _ { f } } \left[\eta^{T}(t)\left(\tilde{A}^{T} P+P \tilde{A}+\tilde{B}_{1}^{T} P \tilde{B}_{1}+\tilde{C}^{T} \tilde{C}\right) \eta(t)+2 \eta^{T}(t) P \tilde{A}_{1} \sigma(\bar{C} Z \eta(t))\right.\right. \\
& \left.\left.+2 \eta^{T}(t) P \tilde{B} v(t)-\gamma^{2} v^{T}(t) v(t)\right] d t\right\} \\
= & \mathbb{E}\left\{\int_{0}^{t_{f}} \xi^{T}(t) \Psi \xi(t) d t\right\}
\end{aligned}
$$

where

$$
\begin{aligned}
\xi(t) & =\left[\begin{array}{lll}
\eta^{T}(t) & \sigma^{T}(\bar{C} Z \eta(t)) & v^{T}(t)
\end{array}\right]^{T}, \\
\Psi & =\left[\begin{array}{ccc}
\tilde{A}^{T} P+P \tilde{A}+\tilde{B_{1}^{T}} P \tilde{B_{1}}+\tilde{C}^{T} \tilde{C} & P \tilde{A_{1}} & P \tilde{B} \\
* & 0 & 0 \\
* & * & -\gamma^{2} I
\end{array}\right] .
\end{aligned}
$$

Similarly, from (13) and (15), we have

$$
\begin{aligned}
& \mathbb{E}\left\{\int_{0}^{t_{f}}\|e(t)\|^{2} d t\right\}-\gamma^{2} \int_{0}^{t_{f}}\|v(t)\|^{2} d t \\
\leq & \mathbb{E}\left\{\int_{0}^{t_{f}}\left\{\xi^{T}(t) \Psi \xi(t)-\varepsilon_{1}[\sigma(\bar{C} Z \eta(t))-\Lambda \bar{C} Z \eta(t)]^{T}[\sigma(\bar{C} Z \eta(t))-\bar{C} Z \eta(t)]\right\} d t\right\} \\
= & \mathbb{E}\left\{\int_{0}^{t_{f}}\left\{\xi^{T}(t) \Phi \xi(t)\right\} d t\right\} \\
< & 0 .
\end{aligned}
$$

for all $\xi(t) \neq 0$. Letting $t_{f} \rightarrow \infty$, the $H_{\infty}$ performance constraint (12) is immediately obtained. The proof of Theorem 1 is therefore complete.

Remark 4: In the proof of Theorem 1, the Itô formula is used to obtain the derivative of the Lyapunov function and sector nonlinear description is employed to characterize the sensor saturation. It should be pointed out that, in the analysis of the $H_{\infty}$ performance of the spatial-temporal systems, the major difficulty results from the complex dynamic behavior induced by spatial-temporal nature. In order to overcome this difficulty, the vector reorganization approach is exploited to transform the spatial-temporal systems into an equivalent ordinary differential dynamic systems. This is the main difference between the methods in this paper and the existing literature and hence the results derived in this paper are not a trivial extension of the existing ones. 
According to the performance analysis conducted in Theorem 1, a solution to the reliable $H_{\infty}$ filtering problem with both sensor saturations and failures is obtained in the following theorem.

Theorem 2: Let the disturbance attenuation level $\gamma>0$ be given. For the spatial-temporal system (1) and sensors with both saturations and failures, the design problem of the reliable $H_{\infty}$ filter is solvable if there exist a positive definite matrix $P=\operatorname{diag}\left\{Q_{1}, Q_{2}\right\}$, a real matrix $Y$ and positive scalars $\varepsilon_{1}$ and $\varepsilon_{2}$ such that

$$
\Xi=\left[\begin{array}{cc}
\Xi_{11} & \Xi_{12} \\
* & \Xi_{22}
\end{array}\right]<0,
$$

where

$$
\begin{aligned}
\Xi_{11} & =\left[\begin{array}{cccc}
\hat{\Xi} & -\bar{H}^{T} \bar{H} & -\varepsilon_{1} \bar{C}^{T}(\Lambda+I) / 2 & Q_{1} \bar{G} \\
-\bar{H}^{T} \bar{H} & \bar{A}^{T} Q_{2}+Q_{2} \bar{A}+\bar{H}^{T} \bar{H} & Y F_{0} & Y F_{0} \bar{D} \\
* & * & -\varepsilon_{1} I & 0 \\
* & * & * & -\gamma^{2} I
\end{array}\right], \\
\hat{\Xi} & =\bar{A}^{T} Q_{1}+Q_{1} \bar{A}+\bar{G}_{1}^{T} Q_{1} \bar{G}_{1}+\bar{H}^{T} \bar{H}-\varepsilon_{1} \bar{C}^{T} \Lambda \bar{C}, \\
\Xi_{12} & =\left[\begin{array}{cccc}
0 & \tilde{F}^{T} Y^{T} & 0 & 0 \\
0 & 0 & \varepsilon_{2} I & \varepsilon_{2} \bar{D}
\end{array}\right]^{T}, \quad \Xi_{22}=\operatorname{diag}\left\{-\varepsilon_{2} I,-\varepsilon_{2} I\right\} .
\end{aligned}
$$

Moreover, if inequality (21) is feasible, the parameter matrix of the desired filter is given as follows:

$$
L_{f}=Q_{2}^{-1} Y
$$

Proof: Noting $P=\operatorname{diag}\left\{Q_{1}, Q_{2}\right\}$ together with (11), we can rewrite $\Phi$ given in Theorem 1 as follows:

$$
\Phi=\left[\begin{array}{cccc}
\hat{\Xi} & -\bar{H}^{T} \bar{H} & -\varepsilon_{1} \bar{C}^{T}(\Lambda+I) / 2 & Q_{1} \bar{G} \\
-\bar{H}^{T} \bar{H} & \bar{A}^{T} Q_{2}+Q_{2} \bar{A}+\bar{H}^{T} \bar{H} & Q_{2} L_{f} F & Q_{2} L_{f} F \bar{D} \\
* & * & -\varepsilon_{1} I & 0 \\
* & * & * & -\gamma^{2} I
\end{array}\right] .
$$

Now, rewrite $F$ as the following form

$$
F=F_{0}+\tilde{F} \chi
$$

with $\chi=\tilde{F}^{-1} \Delta F$ satisfying $\chi \chi^{T} \leq I$. Then, it follows from (24) and (25) that

$$
\Phi=\bar{\Xi}_{11}+\Pi_{1} \chi \Pi_{2}+\Pi_{2}^{T} \chi^{T} \Pi_{1}^{T}
$$

where

$$
\begin{aligned}
\bar{\Xi}_{11} & =\left[\begin{array}{cccc}
\hat{\Xi} & -\bar{H}^{T} \bar{H} & -\varepsilon_{1} \bar{C}^{T}(\Lambda+I) / 2 & Q_{1} \bar{G} \\
-\bar{H}^{T} \bar{H} & \bar{A}^{T} Q_{2}+Q_{2} \bar{A}+\bar{H}^{T} \bar{H} & Q_{2} L_{f} F_{0} & Q_{2} L_{f} F_{0} \bar{D} \\
* & * & -\varepsilon_{1} I & 0 \\
* & * & * & -\gamma^{2} I
\end{array}\right], \\
\Pi_{1} & =\left[\begin{array}{llll}
0 & \tilde{F}^{T} L_{f}^{T} Q_{2}{ }^{T} & 0 & 0
\end{array}\right]^{T}, \quad \Pi_{2}=\left[\begin{array}{llll}
0 & 0 & I & \bar{D}
\end{array}\right] .
\end{aligned}
$$


By using Lemma 1, it can be obtained that $\Phi<0$ if and only if there exists a positive scalar $\varepsilon_{2}$ such that

$$
\bar{\Xi}_{11}+\varepsilon_{2}{ }^{-1} \Pi_{1} \Pi_{1}^{T}+\varepsilon_{2} \Pi_{2}^{T} \Pi_{2}<0 .
$$

In virtue of Schur Complement Lemma and noting the relation $Y=Q_{2} L_{f}$, it is easily seen that (28) is equivalent to $\Xi<0$. This means that the condition of Theorem 1 is satisfied and the rest of proof can be obtained directly from Theorem 1.

Until now, we have analyzed the $H_{\infty}$ performance for the filtering error system with both sensor saturations and failures, and obtained a sufficient condition which ensures the asymptotical stability in probability as well as the $H_{\infty}$ performance requirements. In Theorem 2, the design method of the desired reliable $H_{\infty}$ filter has been given. In the next section, we shall illustrate the effectiveness of the proposed filtering scheme by providing a simulation example.

\section{An Illustrative Example}

In this section, a numerical simulation example is given to show the effectiveness of the filtering methods proposed in this paper.

Given the rectangular region $[0,3] \times[0,3]$, the parameters of the stochastic spatial-temporal system (1) is set as follows

$$
\begin{aligned}
& \kappa=\left[\begin{array}{cc}
6 & 1 \\
0.5 & 3
\end{array}\right], \quad A=\left[\begin{array}{cc}
-2 & -3 \\
1 & 5
\end{array}\right], \quad B=\left[\begin{array}{cc}
-4 & -1 \\
-3 & -2
\end{array}\right], \\
& G=\left[\begin{array}{l}
1 \\
1
\end{array}\right], \quad G_{1}=\left[\begin{array}{ll}
1 & 0 \\
0 & 1
\end{array}\right], \quad H=\left[\begin{array}{ll}
1 & -1
\end{array}\right] \\
& C_{m_{1}, n_{1}}=C_{1,1}=\left[\begin{array}{ll}
0.9 & 0.8
\end{array}\right], \quad D_{m_{1}, n_{1}}=D_{1,1}=0.5 \\
& C_{m_{2}, n_{2}}=C_{2,1}=\left[\begin{array}{ll}
0.7 & 1
\end{array}\right], \quad D_{m_{2}, n_{2}}=D_{2,1}=0.9 \\
& C_{m_{3}, n_{3}}=C_{1,2}=\left[\begin{array}{ll}
0.6 & 0.9
\end{array}\right], \quad D_{m_{3}, n_{3}}=D_{1,2}=1 \\
& C_{m_{4}, n_{4}}=C_{2,2}=\left[\begin{array}{ll}
1.2 & 0.9
\end{array}\right], \quad D_{m_{4}, n_{4}}=D_{2,2}=0.3 .
\end{aligned}
$$

The sensor fault matrix is assumed to be $F=\operatorname{diag}\left\{f_{1}, f_{2}, f_{3}, f_{4}\right\}$ satisfying

$$
\begin{aligned}
& \underline{f}_{1}=0 \leq f_{1} \leq \bar{f}_{1}=0.8, \\
& \underline{f}_{2}=0.3 \leq f_{2} \leq \bar{f}_{2}=0.9, \\
& \underline{f}_{3}=0.3 \leq f_{3} \leq \bar{f}_{3}=0.7, \\
& \underline{f}_{4}=0.4 \leq f_{4} \leq \bar{f}_{4}=1 .
\end{aligned}
$$

Therefore, it is easily obtained that $F_{0}=\operatorname{diag}\{0.4,0.6,0.5,0.7\}$ and $\tilde{F}=\operatorname{diag}\{0.4,0.3,0.2,0.3\}$.

Moreover, the saturation parameters are set as $\Lambda=\operatorname{diag}\{0.5,0.6,0.4,0.6\}$, the disturbance attenuation level is selected as $\gamma=0.25$, the boundary condition is taken as $x_{0,0}(t)=x_{0,3}(t)=x_{3,0}(t)=x_{3,3}(t)=0$ and the 
initial conditions at the inner points are chosen as

$x_{1,1}^{0}=x_{1,2}^{0}=x_{2,1}^{0}=x_{2,2}^{0}=\left[3 \times e^{-10 \times|0.06-0.03 m|} \times e^{-30 \times|0.06-0.03 n|} \quad 2 \times e^{-10 \times|0.06-0.03 m|} \times e^{-30 \times|0.06-0.03 n|}\right]^{T}$.

With the above parameters, we solve (21) by using LMI toolbox in Matlab and the filter gain matrix $L_{f}$ can be obtained as follows

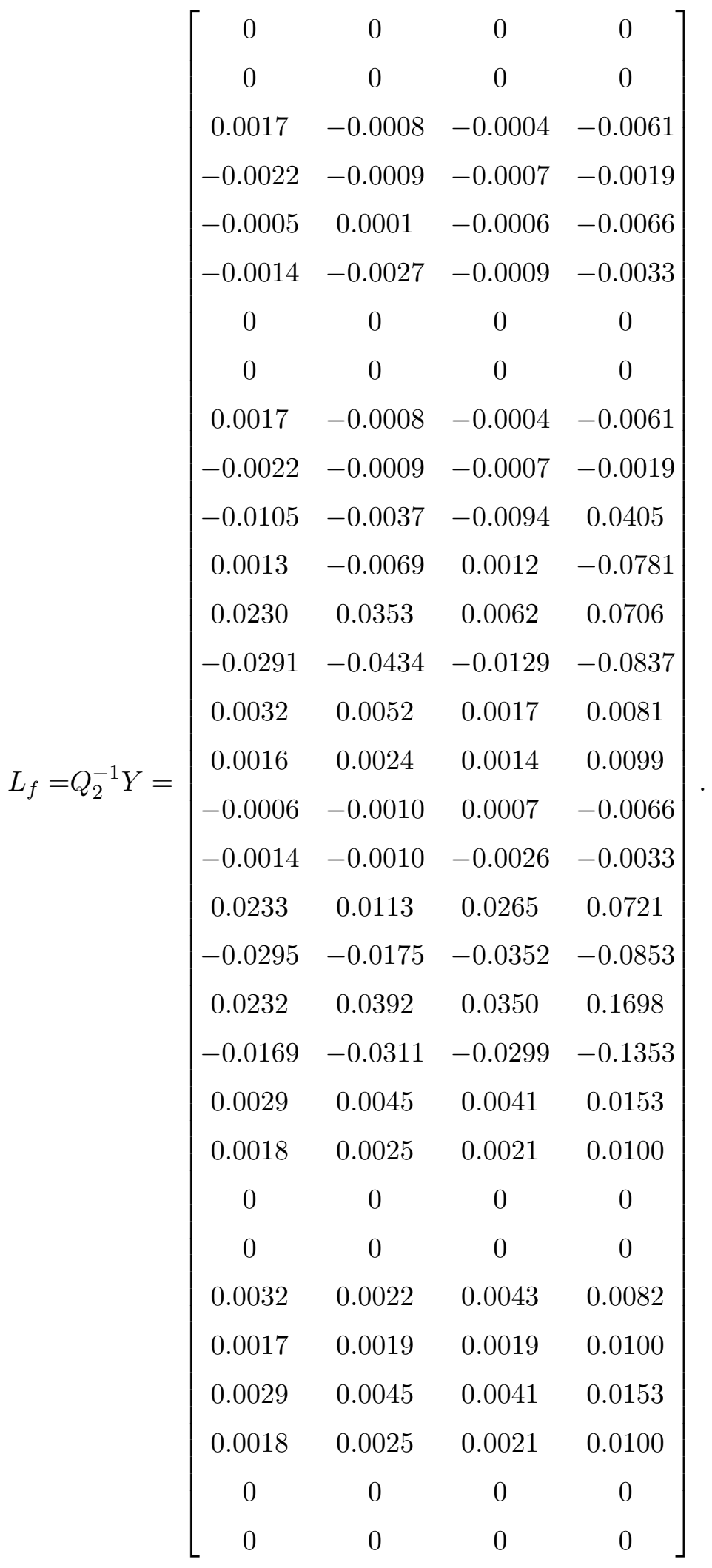


In the simulation, the external disturbance is taken as $v_{m, n}(t)=\sin (0.2 t) \times e^{-0.2 t-0.003 m}$ and the sensor fault matrix is chosen as $F=\operatorname{diag}\{0.75,0.8,0.6,0.9\}$. The simulation results are shown in Figs. 1-8. Figs. 1-4 plot the actual system outputs and their outputs at all inner points. Figs. 5-8 depict the their filtering errors. The simulation results have demonstrated the effectiveness of the proposed reliable $H_{\infty}$ filter.

\section{Conclusions}

In this paper, we have studied the reliable $H_{\infty}$ filtering problem for a class of stochastic spatial-temporal systems with sensor saturations and failures. The dynamic behavior of systems under consideration evolves in both space and time. In order to handle the sensor failures, a reliable $H_{\infty}$ filtering scheme has been proposed for the stochastic spatial-temporal system. By using the vector reorganization approach and Lyapunov stability theory, a sufficient condition has been derived under which the filtering error dynamics is asymptotically stable in probability and the $H_{\infty}$ performance requirement is satisfied. Then, the design method of the desired reliable $H_{\infty}$ filter has been given. Finally, a simulation example has been presented to show the effectiveness of the filtering scheme proposed.

\section{REFERENCES}

[1] B. Shen, Z. Wang, H. Shu and G. Wei, On nonlinear $H_{\infty}$ filtering for discrete-time stochastic systems with missing measurements, IEEE Transactions on Automatic Control, Vol. 53, No. 9, pp. 2170-2180, Oct. 2008.

[2] N. Bar Am and E. Fridman, Network-based $H_{\infty}$ filtering of parabolic systems, Automatica, Vol. 50, No. 12, pp. 3139-3146, Dec. 2014.

[3] M. Chadli, A. Abdo and S. X. Ding, $H_{-} / H_{\infty}$ fault detection filter design for discrete-time Takagi-Sugeno fuzzy system, Automatica, Vol. 49, No. 7, pp. 1996-2005, Jul. 2013.

[4] D. Ding, Z. Wang, B. Shen and H. Dong, Envelope-constrained $H_{\infty}$ filtering with fading measurements and randomly occurring nonlinearities: the finite horizon case, Automatica, Vol. 55, pp. 37-45, May. 2015.

[5] E. Gershon, U. Shaked and I. Yaesh, $H_{\infty}$ control and filltering of discrete-time stochastic systems with multiplicative noise, Automatica, Vol. 37, No. 3, pp. 409-417, Mar. 2001.

[6] Y. Hung and F. Yang, Robust $H_{\infty}$ filtering with error variance constraints for discrete time-varying systems with uncertainty, Automatica, Vol. 39, No. 7, pp. 1185-1194, Jul. 2003.

[7] H. Li and M. Fu, A linear matrix inequality approach to robust $H_{\infty}$ filtering, IEEE Transactions on Signal Processing, Vol. 45, No. 9, pp. 2338-2350, Sep. 1997.

[8] J. Liang, F. Sun and X. Liu, Finite-horizon $H_{\infty}$ filtering for time-varying delay systems with randomly varying nonlinearities and sensor saturations, Systems Science and Control Engineering: An Open Access Journal, Vol. 2, No. 1, pp. 108-118, 2014.

[9] Y. Song, G. Wei and G. Yang, Distributed $H_{\infty}$ filtering for a class of sensor networks with uncertain rates of packet losses, Signal Processing, Vol. 104, pp. 143-151, Nov. 2014.

[10] L. Wu, P. Shi, H. Gao and C. Wang, $H_{\infty}$ filtering for 2-D Markovian jump systems, Automatica, Vol. 44, No. 7, pp. 1849-1858, Jul. 2008.

[11] W. Zhang, Y. Huang and H. Zhang, Stochastic $H_{2} / H_{\infty}$ control for discrete-time systems with state and disturbance dependent noise, Automatica, Vol. 43, pp. 513-521, 2007.

[12] D. Zhang, Q. Wang, L. Yu and Q. Shao, $H_{\infty}$ filtering for networked systems with multiple time-varying transmissions and random packet dropouts, IEEE Transactions on Industrial Informatics, Vol. 9, No. 3, pp. 1705-1716, Aug. 2013.

[13] W. Zhang, H. Dong, G. Guo and L. Yu, Distributed sampled-data $H_{\infty}$ filtering for sensor networks with nonuniform sampling periods, IEEE Transactions on Industrial Information, Vol. 10, No. 2, pp. 871-881, May. 2014. 
[14] Q. Zhong, J. Cheng, Y. Zhao, J. Ma and B. Huang, Finite-time $H_{\infty}$ filtering for a class of discrete-time Markovian jump systems with switching transition probabilities subject to average dwell time switching, Applied Mathematics and Computation, Vol. 225, pp. 278-294, Dec. 2013.

[15] D. Ding, Z. Wang, B. Shen and H. Shu, $H_{\infty}$ state estimation for discrete-time complex networks with randomly occurring sensor saturations and randomly varying sensor delays, IEEE Transactions on Neural Networks and Learning Systems, Vol. 23, No. 5, pp. 725-736, May. 2012.

[16] H. Dong, Z. Wang, D. W. C. Ho and H. Gao, Robust $H_{\infty}$ filtering for Markovian jump systems with randomly occurring nonlinearities and sensor saturation: the finite-horizon case, IEEE Transactions on Signal Processing, Vol. 59, No. 7, pp. 30483057, Jul. 2011.

[17] T. Hu, Z. Lin and B. Chen, Analysis and design for discrete-time linear systems subject to actuator saturation, Systems 8 Control Letters, Vol. 45, No. 2, pp. 97-112, Feb. 2002.

[18] Z. Wang, B. Shen and X. Liu, $H_{\infty}$ filtering with randomly occurring sensor saturations and missing measurements, Automatica, Vol. 48, No. 3, pp. 556-562, Mar. 2012.

[19] Y. Xiao, Y. Cao and Z. Lin, Robust filtering for discrete-time systems with saturation and its application to transmultiplexers, IEEE Trans. Signal Processing, Vol. 52, No. 5, pp. 1266-1277, May. 2004.

[20] F. Yang and Y. Li, Set-membership filtering for systems with sensor saturation, Automatica, Vol. 45, No. 8, pp. 1896-1902, Aug. 2009.

[21] W. Yang, M. Liu and P. Shi, $H_{\infty}$ filtering for nonlinear stochastic systems with sensor saturation, quantization and random packet losses, Signal Processing, Vol. 92, No. 6, pp. 1387-1396, Jun. 2012.

[22] Z. Zuo, D. W. C. Ho and Y. Wang, Fault tolerant control for singular systems with actuator saturation and nonlinear perturbation, Automatica, Vol. 46, No. 3, pp. 569-576, Mar. 2010.

[23] E. Tian and D. Yue, Reliable $H_{\infty}$ filter design for T-S fuzzy model-based networked control systems with random sensor failure, International Journal of Robust and Nonlinear control. Vol. 23, No. 1, pp. 15-32, Jan. 2013.

[24] G. Yang and D. Ye, Adaptive reliable $H_{\infty}$ filtering against sensor failures, IEEE Transactions on Signal Processing, Vol. 55, No. 7, pp. 3161-3171, Jul. 2007.

[25] G. Wei, F. Han, L. Wang and Y. Song, Reliable $H_{\infty}$ filtering for discrete piecewise linear systems with infinite distributed delays, International Journal of General Systems, Vol. 43, No. 3-4, pp. 346-358, May. 2014.

[26] W. Chen and B. Chen, Robust filter for linear stochastic partial differential systems via a set of sensor measurements, IEEE Transactions Circuits and Systems -I: Regular Papers, Vol. 59, No. 6, pp. 1251-1264, Jun. 2012.

[27] B. Chen, W. Chen and W. Zhang, Robust filter for nonlinear stochastic partial differential systems in sensor signal processing: fuzzy approach, IEEE Transactions on Fuzzy Systems, Vol. 20, No. 5, pp. 957-970, Oct. 2012.

[28] U. A. Khan and J. M. F. Moura,Distributing the Kalman filter for large-scale systems, IEEE Transactions on Signal Processing, Vol. 56, No. 10, pp. 4919-4935, Oct. 2008.

[29] R. Hasminskii, Stochastic Stability of Differential Equations, Alphen, The Netherlands: Sijtjoff and Noordhoff, 1980.

[30] S. Boyd, L. Ghaoui, E. Feron and V. Balakrishnan, Linear Matrix Inequalities in System and Control Theory, SIAM: philadelphia, 1994. 


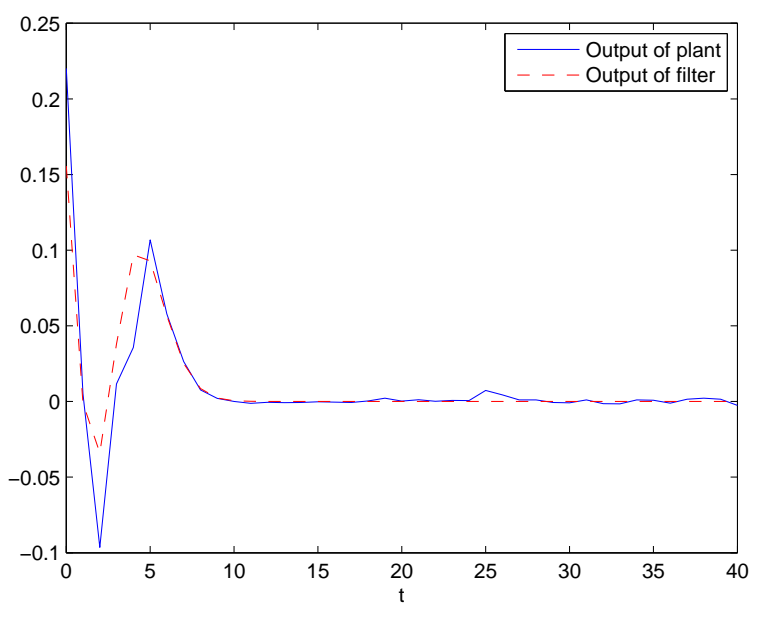

Fig. 1. Output $z_{11}(t)$ and its estimate $\hat{z}_{11}(t)$.

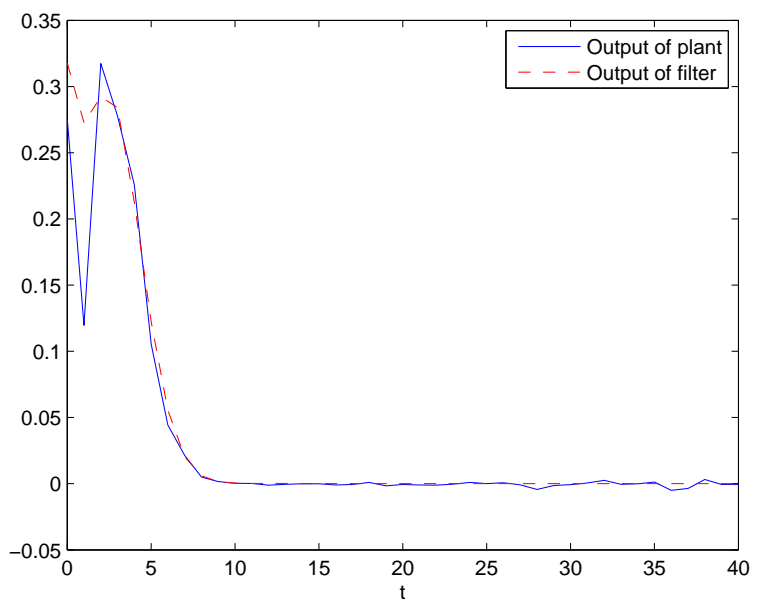

Fig. 3. Output $z_{21}(t)$ and its estimate $\hat{z}_{21}(t)$.

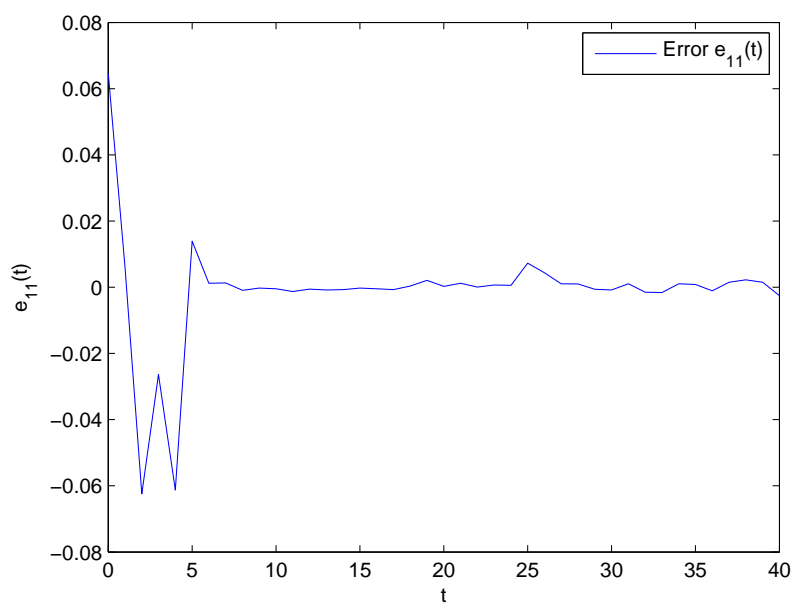

Fig. 5. Filtering error $e_{11}(t)$.

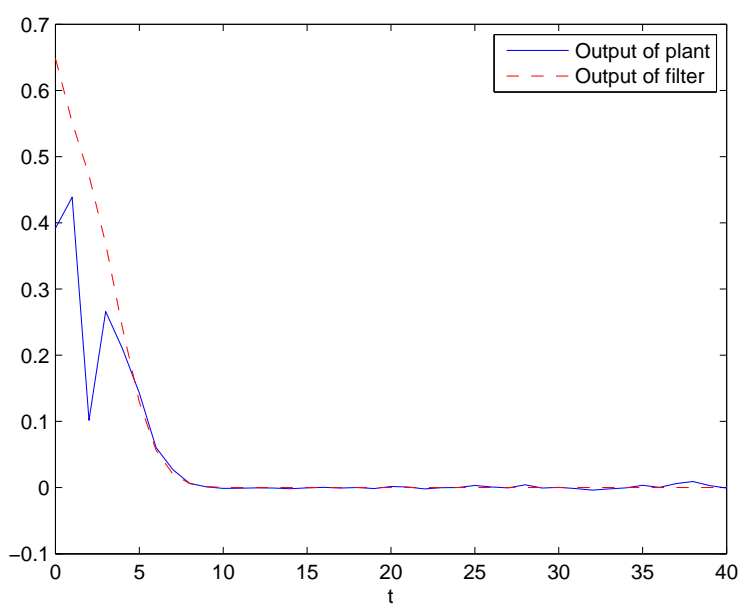

Fig. 2. Output $z_{12}(t)$ and its estimate $\hat{z}_{12}(t)$.

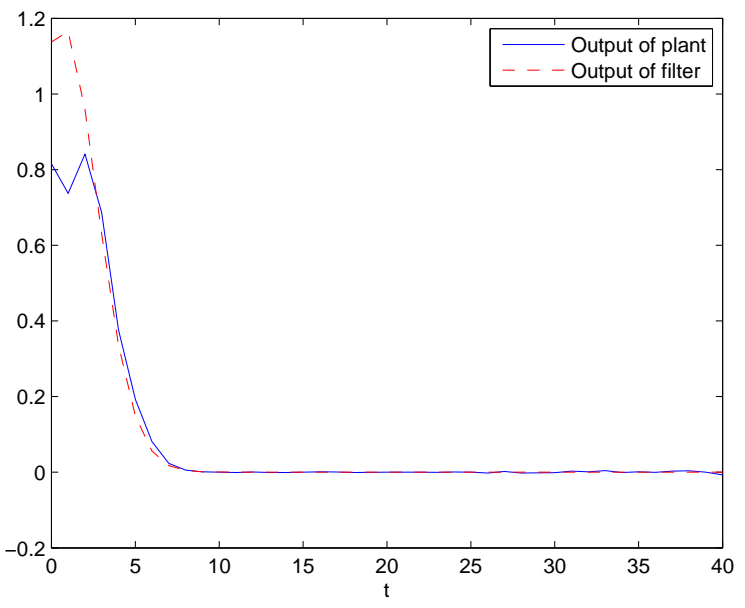

Fig. 4. Output $z_{22}(t)$ and its estimate $\hat{z}_{22}(t)$.

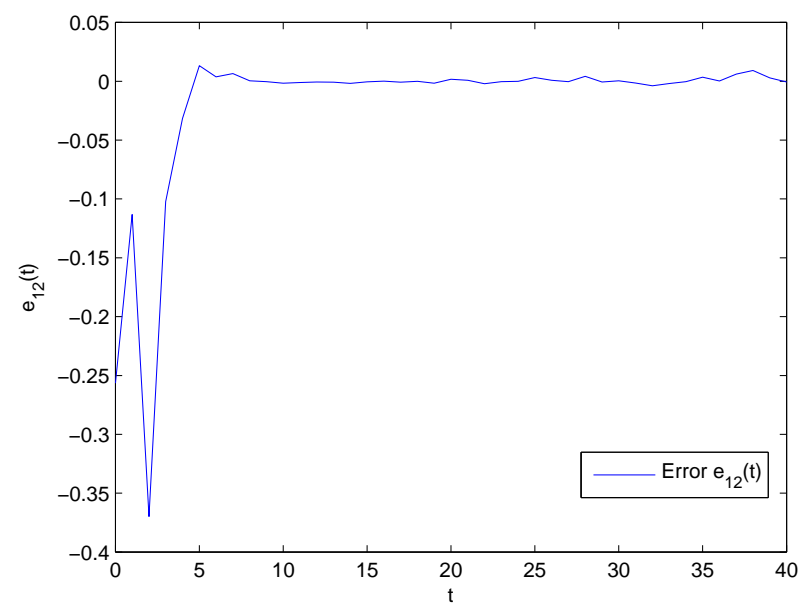

Fig. 6. Filtering error $e_{12}(t)$. 


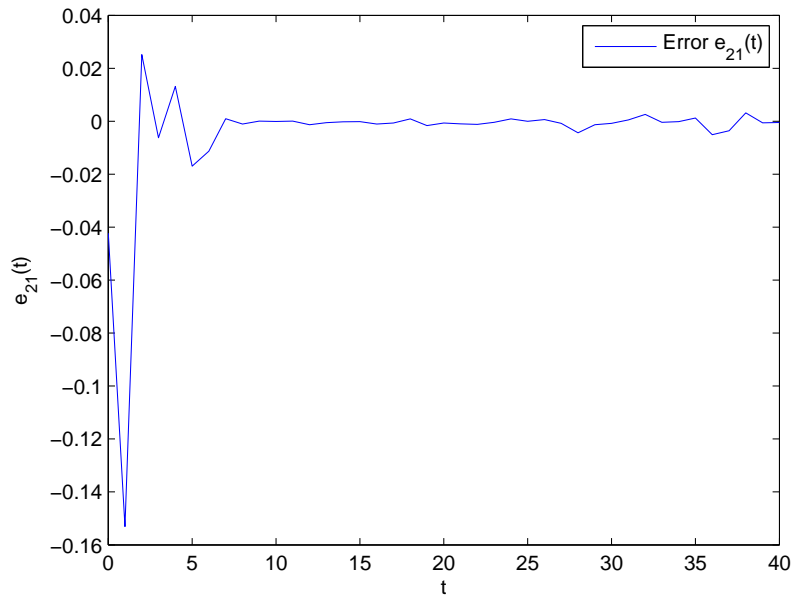

Fig. 7. Filtering error $e_{21}(t)$.

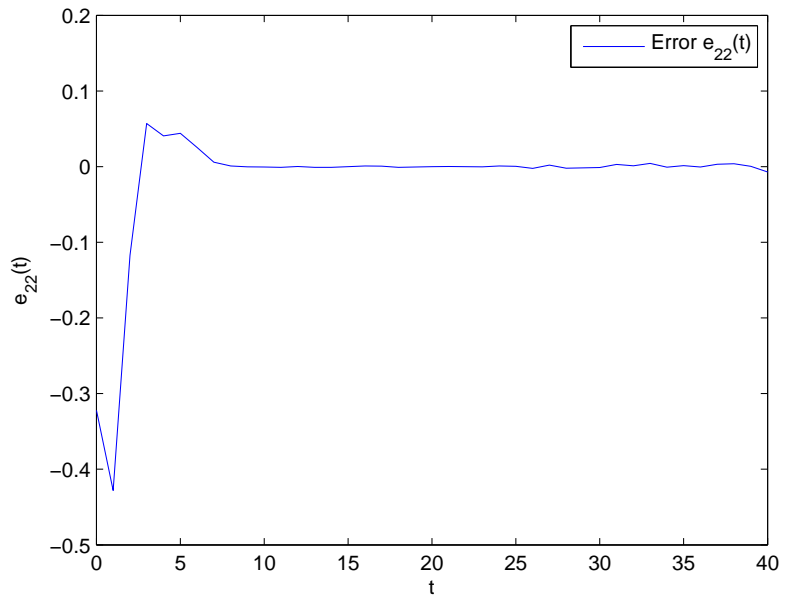

Fig. 8. Filtering error $e_{22}(t)$. 